\title{
NRAS Protein Variant
}

National Cancer Institute

\section{Source}

National Cancer Institute. NRAS Protein Variant. NCI Thesaurus. Code C98439.

A variation in the amino acid sequence for the GT Pase NRas protein. 